\title{
VARIABILIDAD MORFOLÓgICA DE RUPRECHTIA APETALA WEDD. (POLYGONACEAE) EN LAS SIERRAS PAMPEANAS Y SUBANDINAS DE LA ARgENTINA
}

\author{
MoRPHOLOgICAL VARIABILITY OF RUPRECHTIA APETALA WEDD. \\ (Polygonaceae) In the subandean AND pampean sierRas of Argentina
}

\author{
Ángeles Tessore ${ }^{1^{*} \mathbb{D}}$, Virginia Y. Mogni ${ }^{1} \mathbb{D}$, Luis J. Oakley ${ }^{1}$ y Darién E. Prado ${ }^{1,2} \mathbb{D}$
}

1. Botánica, Facultad de Ciencias Agrarias, Universidad Nacional de Rosario, C.C. №14, 2125 Zavalla, Argentina.

2. Instituto de Investigaciones en Ciencias Agrarias, IICAR CONICETUNR, 2125 Zavalla, Argentina.

*angelestessore@gmail.com

Citar este artículo

TESSORE, Á., V. Y. MOGNI, L. J. OAKLEY y D. E. PRADO. 2020 Variabilidad morfológica de Ruprechtia apetala wedd. (Polygonaceae) en las sierras pampeanas y subandinas de la Argentina. Bol. Soc. Argent. Bot. 55: 389-401.

DOI: https://doi. org/10.31055/1851.2372.v55. n3.26175

Recibido: 28 Noviembre 2019 Aceptado: 28 Julio 2020 Publicado: 30 Septiembre 2020 Editor: Roberto Manuel Salas (D)

\section{SUMMARY}

Background and aims: Ruprechtia apetala (Polygonaceae) is a woody Chaquenian species with large morphological variability throughout its distribution range. The present study attempts to evaluate this variability within Argentina.

M\&M: Forty-two individuals were collected from six Argentinian provinces, corresponding to the regions of Sierra Chaco, Piedmont Forest and the transition zone between them. Numerous morphological characters, both reproductive and vegetative, were analyzed. Principal Components and Conglomerates Analysis were performed, in order to establish the existence of morphogroups; the latter were evaluated by univariate analysis (Kruskal-Wallis test and cluster description by $\mathrm{R}$ ) and multivariate (ANOSIM and PERMANOVA), to detect possible significant intraspecific differences. Additionally, a distribution map of the collected specimens was drawn up.

Results: From the multivariate analysis two morphological groups were detected, mainly at foliar and growth habit levels. The ANOSIM and PERMANOVA analysis show a significant global difference among both groups ( $p$-value $<0,05$ ); however, the corresponding statistics indicate a high similarity between them. Additionally, it was demonstrated that no characters are mutually exclusive to either group.

Conclusions: Observed morphological differences between specimens from different biogeographical regions, though not mutually exclusive, allow postulating the existence of ecotypes within the species.

\section{KEY WORDS}

Chaco, ecotypes, morphological variables, Seasonally Dry Forests.

\section{RESUMEN}

Introducción y Objetivos: Ruprechtia apetala (Polygonaceae) es una especie que presenta una gran variabilidad morfológica entre individuos a lo largo de su rango de distribución. El presente estudio tiene como objetivo evaluar dicha variabilidad morfológica dentro del territorio argentino.

M\&M: se colectaron 42 individuos en seis provincias argentinas correspondientes a las regiones Chaqueña serrana, Selva Pedemontana y a la zona de transición entre ellas. Fueron analizados numerosos caracteres morfológicos, tanto vegetativos como reproductivos. Se realizaron Análisis de Componentes Principales y de Conglomerados para detectar morfogrupos; estos últimos se evaluaron mediante análisis univariados (Prueba de Kruskal-Wallis y descripción de clusters mediante R) y multivariados (ANOSIM y PERMANOVA), para evaluar posibles diferencias intraespecíficas significativas. Adicionalmente, se confeccionó un mapa de distribución biogeográfica de los individuos.

Resultados: a partir del análisis multivariado se detectaron dos grupos morfológicos, principalmente a niveles foliar y de hábito de crecimiento. Los Análisis ANOSIM y PERMANOVA muestran que existe una diferencia global significativa entre ambos grupos ( $p$-valor $<0,05$ ); no obstante, los estadísticos correspondientes indican una elevada similitud entre ellos. Adicionalmente, mediante la descripción de cada conglomerado, se corroboró que no existen estados de caracteres mutuamente excluyentes para uno $u$ otro grupo.

Conclusiones: La variabilidad morfológica encontrada entre ejemplares de distintas regiones biogeográficas, y la no exclusividad de caracteres morfológicos para cada grupo particular, permite postular la existencia de ecotipos en la especie.

\section{Palabras Clave}

Bosques estacionalmente secos, Chaco, ecotipos, variables morfológicas. 


\section{INTRODUCCIÓN}

\section{Generalidades y descripción}

El género Ruprechtia C.A. Mey. (Polygonaceae) es exclusivamente americano y presenta un área de distribución continua, entre $\operatorname{los} 26^{\circ}$ lat. $\mathrm{N}$ y $\operatorname{los} 35^{\circ}$ lat. S (Cocucci, 1958). En la Argentina, el mayor número de especies se halla en el norte del país, entre las cuales Ruprechtia apetala Wedd. es una de las que alcanzan la distribución más austral, desde el sur de Bolivia hasta las últimas estribaciones de las Sierras Pampeanas en Córdoba (Cocucci, 1958). En esta última región se la conoce como "manzano del campo" debido a que las hojas son similares a las del manzano común (Malus sp.) (Demaio et al., 2002). Otros nombres vernáculos que se le atribuyen son "duraznillo", "duraznillo blanco", "higuerón", "higuerilla hembra", "higuerilla macho", "marmelero", "sacha membrillo", "virarú", "virarú colorado" (Cocucci, 1958; de la Peña \& Pensiero, 2004).

Esta especie comprende árboles y arbustos dioicos, de entre 2 y $12 \mathrm{~m}$ de altura y un tronco de hasta 20-30 cm de diámetro (Cocucci, 1958), el que se caracteriza por su corteza grisácea, lisa y persistente. Las ramas son nudosas y lenticeladas, las más jóvenes pubescentes y provistas de ócreas caducas en los nudos (Digilio \& Legname, 1966). Las hojas son caducas, alternas y simples, elípticas u oblongas, de borde crenado u ondulado, coriáceas, pubescentes (principalmente en el envés), ásperas al tacto, cordadas u obtusas en la base, de ápice agudo y a veces acuminado. La lámina presenta dimensiones variables, de 2,5 a $12,5 \mathrm{~cm}$ de largo y de 1,5 a $7 \mathrm{~cm}$ de ancho, con nervaduras impresas en el haz y prominentes en el envés. El pecíolo es pubescente y cilíndrico, provisto de una ócrea membranácea (Cocucci, 1961; Digilio \& Legname, 1966; Demaio et al., 2002). Las inflorescencias son racimos compuestos axilares o terminales (Cocucci, 1958, 1961). Las flores son pediceladas (Cialdella \& Brandbyge, 2001) y manifiestan un marcado dimorfismo sexual, siendo las estaminadas de coloraciones amarillo-verdosas o rosado-pálidas, con tres sépalos membranáceos, libres y obtusos, externamente pubescentes, que alternan con tres pétalos a los cuales se les asimilan en forma y tamaño. Las flores pistiladas, en cambio, presentan piezas periánticas rojizas, purpúreas, rosadas o amarillentas, con los sépalos unidos en la base en un breve tubo y externamente pubescentes, mientras que los pétalos son pequeños, deltoides y vestigiales, generalmente atrofiados (Cocucci, 1958, 1961; Digilio \& Legname, 1966; Cialdella \& Brandbyge, 2001; Pendry, 2004). El fruto es un aquenio con un pericarpio muy delgado, glabro, trilobulado, protegido por el cáliz persistente con sépalos prolongados en forma de alas (Cocucci, 1958); éstos pueden presentar tonalidades castañas o rojizas (Demaio et al., 2002), aunque también presentan coloraciones pálidas, rosadas, o castaño anaranjadas (Pendry, 2004). La semilla ocupa prácticamente todo el interior del fruto, y es más o menos piriforme, tri-lobulada (Cocucci, 1958, 1961).

En cuanto a la fenología, la floración ocurre de diciembre a enero y la fructificación desde febrero a abril (Digilio \& Legname, 1966).

\section{Historia taxonómica de Ruprechtia apetala}

Ruprechtia apetala fue descripta por Weddell (1849) basándose en material colectado por este autor en Bolivia, en cercanías del río Pilcomayo (dpto. Chuquisaca). Este autor también describió una especie afín, R. mollis Wedd., con base en un ejemplar colectado en el mismo país. Años después, Grisebach (1874) describió $R$. corylifolia Griseb. basándose en un ejemplar colectado en las estribaciones de la Sierra de Ascochinga (Córdoba), y R. excelsa Griseb. con base en material proveniente de la provincia de Tucumán; y a principios del siglo pasado Herzog (1922) describió R. boliviensis Herzog a partir de un ejemplar boliviano. Posteriormente, Cocucci (1958) consideró que $R$. excelsa y $R$. boliviensis eran sinónimos de $R$. corylifolia, debido a la similitud que observó tanto en sus caracteres vegetativos como en los reproductivos. Este mismo autor (Cocucci, 1961), en su monografía del género Ruprechtia, incluyó en la sinonimia de $R$. apetala a los taxones mencionados y también a $R$. mollis, decisión válida de acuerdo al artículo 11.5 del ICBN (Turland et al., 2018). Para esto, se basó en la importancia taxonómica de la ausencia de pétalos en las flores femeninas.

\section{Área de distribución}

Ruprechtia apetala se extiende desde el sur de Bolivia hasta el centro de la Argentina, 


\section{Á. Tessore et al. - Variabilidad morfológica de Ruprechtia apetala en Argentina}

donde habita la zona que abarcan las Sierras Subandinas al norte y las Sierras Pampeanas al sur, atravesando las provincias de Jujuy, Salta, Tucumán, Catamarca, Santiago del Estero, La Rioja, Córdoba y extremo norte de San Luis (Cocucci, 1958; Pendry, 2004) (Fig. 1). También se han registrado especímenes en Paraguay, en el departamento Chaco -parte del actual Alto Paraguay- (Cialdella \& Brandbyge, 2001). Es considerada como una especie orófila, especialmente abundante en el Chaco Serrano y en la transición de éste con sus ambientes aledaños (Cabido et al., 1991; Demaio et al., 2002). Asimismo, habita en bosques secos caducifolios, en laderas y bordes de ríos, entre los 100 y 2000 $\mathrm{m}$ de altitud (Morales et al., 1995; Pendry, 2004). Estas áreas se corresponden con las unidades: "Selva pluvial semicaducifolia pedemontanaSelva de Transición”, "Bosque de xerófitas con Schinopsis marginata-Chaco Serrano" y "Bosque de xerófitas con Schinopsis lorentziiChaco Semiárido", descriptas por Oyarzabal et al. (2018).

Empíricamente, se ha observado tanto a campo como en ejemplares de herbario que $R$. apetala presenta una gran variabilidad morfológica entre individuos de las distintas regiones de distribución en nuestro país. La experiencia de campo indica que existen algunas diferencias importantes entre sus poblaciones: desde arbustos de 2-3 $\mathrm{m}$ de altura en los sectores más secos y pedregosos del centrooeste de Argentina (Cocucci, 1958), hasta árboles de unos $10 \mathrm{~m}$ en los bosques secos estacionales del noroeste, sobre suelos más fértiles y mayores

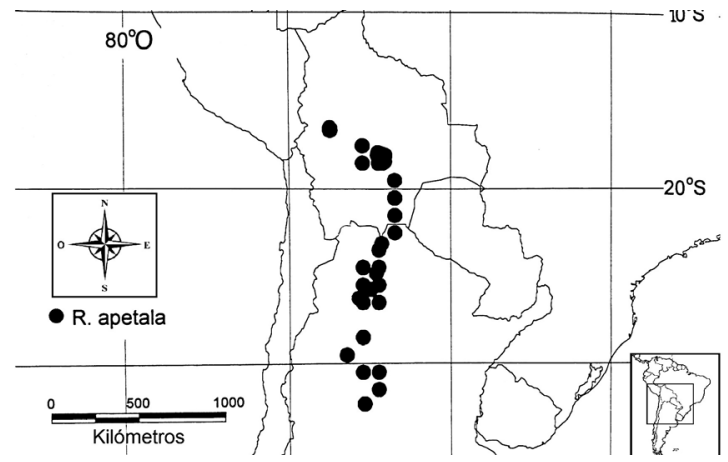

Fig. 1. Área de distribución de R. apetala. Modificado de Pendry (2004). precipitaciones (Prado, 1995) (Fig. 2). También se han observado diferencias marcadas respecto a algunos caracteres morfológicos, como el tamaño, forma y consistencia de las hojas y el tamaño de flores y frutos. Por lo tanto, el presente trabajo tiene como objetivo estudiar la variabilidad de los caracteres morfológicos de Ruprechtia apetala a lo largo de gran parte de su rango de distribución en la Argentina.
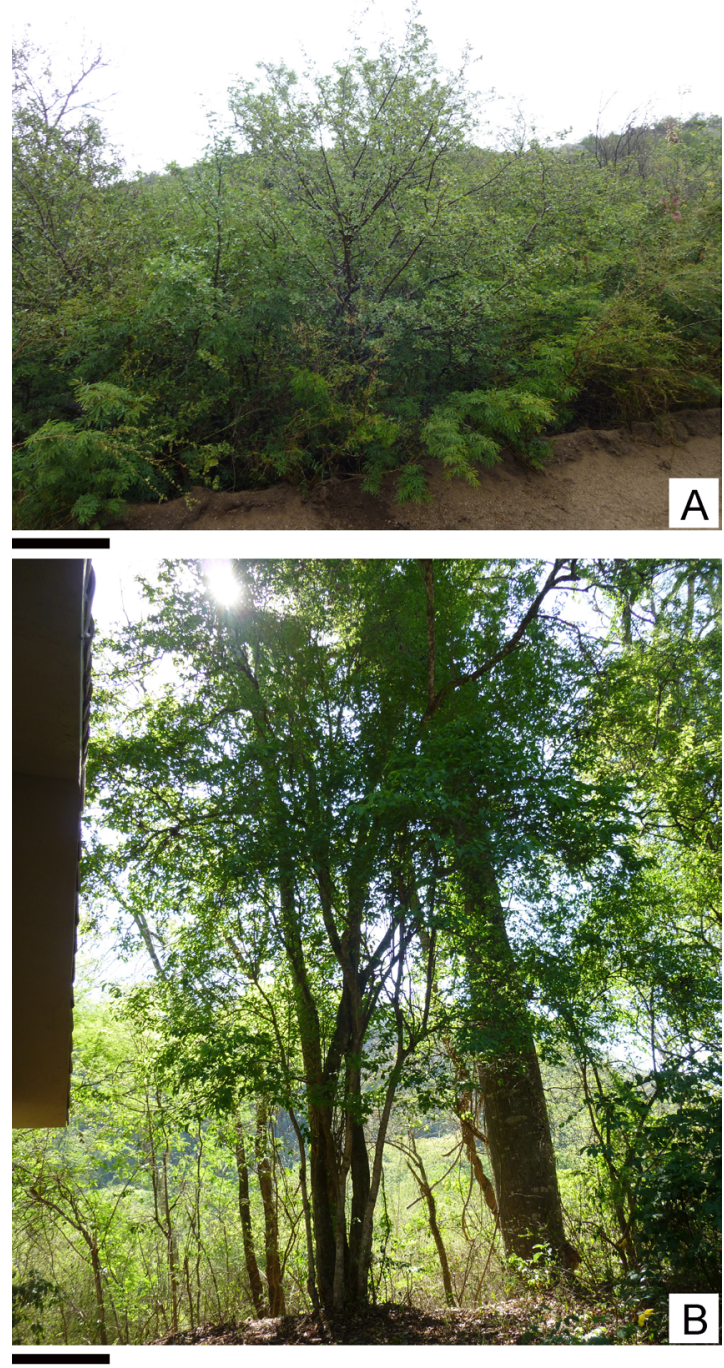

Fig. 2. Individuos de $R$. apetala en distintos ambientes. A: Ejemplar de hábito arbustivo, provincia de Córdoba. B: Ejemplar de hábito arbóreo, provincia de Jujuy. Escalas= $A$ : $1 \mathrm{~m}$; $B$ : $0,5 \mathrm{~m}$. 


\section{Materiales y Métodos}

Con el objetivo de colectar muestras de numerosos especímenes de $R$. apetala y, al mismo tiempo, tratar de cubrir la variabilidad existente a lo largo de todo el área de distribución de la especie en Argentina, se realizó un viaje de campaña durante los meses de noviembre y diciembre de 2014. Se colectaron 42 especímenes (dos ramas de la copa de cada uno: una basal y otra superior, representativas de la variabilidad observada en el individuo) provenientes de las provincias de Córdoba, Santiago del Estero, Catamarca, Tucumán, Salta y Jujuy (ver Material Suplementario 1a- Especímenes colectados). Es preciso aclarar que al momento de realizarse las colectas, la mayoría de los ejemplares no se encontraban en estado reproductivo, o bien conservaban sólo restos de inflorescencias o frutos ya senescentes. Adicionalmente se consultaron colecciones disponibles en bases de datos (http:// www.tropicos.org, Missouri Botanical Garden, y http://www.plants.jstor.org, Jstor Global Plants -ejemplares tipo-), para evaluar especímenes identificados como $R$. apetala provenientes de otros puntos del país y de Bolivia, complementando el material colectado en el campo y poder así ajustar la descripción botánica. También se consultaron las colecciones de $R$. apetala depositadas en el Herbario Dra. Carmen L. Cristóbal -IBONE(CTES) y en el Herbario del Instituto Miguel Lillo de Tucumán (LIL), para ampliar la descripción botánica de la especie (ver Material Suplementario 1b- Material adicional examinado).

Para el análisis morfológico se emplearon un total de 35 caracteres vegetativos y 32 reproductivos de los individuos colectados (ver Material Suplementario-Tablas 1a y 1b), utilizando el programa morfométrico tpsDig2 (Rohlf, 2013) y una lupa estereoscópica. Los datos obtenidos fueron procesados utilizando el programa estadístico Infostat (Di Rienzo et al., 2013). Para esto fue necesaria una previa edición y acondicionamiento de los datos. En primer lugar, las variables cualitativas fueron transformadas mediante la creación de variables dummy o variables auxiliares (Infostat, 2004) (ver Material Suplementario-Tabla 1a) y todos los datos fueron estandarizados para contar con una medida estándar de aquellos que tuviesen distintas unidades de medición. Posteriormente se procedió a realizar el análisis estadístico a fin de evaluar la variabilidad morfológica del material. Para ello se seleccionaron 25 variables correspondientes sólo a los caracteres vegetativos (ver Material Suplementario-Tabla 1a) y discriminando a los reproductivos, debido a la cantidad de datos faltantes para estos últimos ( $\sim 60 \%$ de los individuos no presentó flor y/o fruto al momento de la colecta). Utilizando Infostat (Di Rienzo et al., 2013) se realizaron Análisis de Componentes Principales (ACP), Análisis de Conglomerados (AC) y Análisis de la Varianza no Parámetricos (Prueba de Kruskall-Wallis). Con el programa estadístico PAST (Hammer et al., 2001) se realizaron Análisis de la Varianza Multivariado con Permutaciones (PERMANOVA) y Análisis de Similitud (ANOSIM). Por otra parte, se realizó la descripción de los clusters mediante $\mathrm{R}$ (R Core Team, 2015) utilizando el paquete estadístico FactoMineR (Lê et al., 2008). Adicionalmente, los 42 puntos de colecta fueron mapeados utilizando el programa DIVA-GIS (Hijmans et al., 2007), a fin de visualizar la distribución de los mismos.

En todos los casos se priorizó el análisis sobre material de colecta propia por poseer estructuras frescas o mejor conservadas, sumado a la certeza de la procedencia de las ramas según su ubicación en la copa y por los datos disponibles de cada individuo en particular. No obstante, los ejemplares adicionales examinados (ver Material Suplementario 1b- Material adicional examinado) fueron considerados para ajustar la descripción botánica de la especie.

\section{Resultados}

En el ACP se retuvieron 24 componentes principales (CP), las cuales acumulan el $91 \%$ de la variabilidad observada. La CP1 y la CP2 explican en conjunto sólo el $20 \%$ de la variabilidad total. Las variables más correlacionadas a la CP1 (valor de correlación $>|0,7|$ ) son: longitud de lámina, longitud de hoja y ángulo de divergencia de nervaduras secundarias; mientras que para la $\mathrm{CP} 2$ (valor de correlación $>|0,6|$ ) son: prominencia de nervaduras en envés, ancho de pecíolo y ancho de hoja (Tabla 1).

En el biplot resultante (Fig. 3) se puede observar que las CP1 y CP2 ordenan -hacia los cuadrantes derechos del gráfico- a los individuos 


\section{Á. Tessore et al. - Variabilidad morfológica de Ruprechtia apetala en Argentina}

Tabla 1. Variables morfológicas vegetativas más correlacionadas a la CP1 (valor de correlación $>|0,7|$ ) y a la CP2 (valor de correlación>|0,6|).

\begin{tabular}{|lccc|}
\hline \multicolumn{1}{|c}{ Variables } & Abreviaturas & CP 1 & CP 2 \\
\hline Longitud de hoja & LongH & $\mathbf{0 . 7 8}$ & -0.42 \\
\hline Ancho de hoja & AnchoH & 0.62 & $-\mathbf{0 . 6}$ \\
Longitud de lámina & LongLamH & $\mathbf{0 . 7 9}$ & -0.41 \\
Ancho de pecíolo & AnchoPecH & 0.44 & $-\mathbf{0 . 6 1}$ \\
$\begin{array}{l}\text { Prominencia de nervaduras en envés (poco prominentes) } \\
\text { Prominencia de nervaduras en envés (prominentes) }\end{array}$ & NEH_1 & 0.13 & $\mathbf{0 . 6 3}$ \\
$\begin{array}{l}\text { Ángulo de divergencia de nervaduras } \\
\text { secundarias (cercano a 90-abiertas) }\end{array}$ & NEH_2 & -0.13 & $-\mathbf{0 . 6 3}$ \\
$\begin{array}{l}\text { Ángulo de divergencia de nervaduras } \\
\text { secundarias (agudo-cerradas) }\end{array}$ & DiNH_1 & $-\mathbf{0 . 7}$ & -0.15 \\
\hline
\end{tabular}

que presentan valores más altos para las variables longitud de hoja (Long.H), longitud de lámina (Long.Lam.H) y ancho de hoja (Ancho.H), así como también un hábito de crecimiento predominantemente arbóreo (HábitoCrec_1), hojas generalmente membranáceas ( $\mathrm{CH}_{-} 2$ ), elíptico-lanceoladas (FH_4) y ángulo agudo de divergencia de nervaduras secundarias (DiNH_2). Contrariamente, los individuos ubicados en los cuadrantes opuestos, a la izquierda del biplot, presentan menores dimensiones de hoja y lámina, generalmente hábito de crecimiento arbustivo

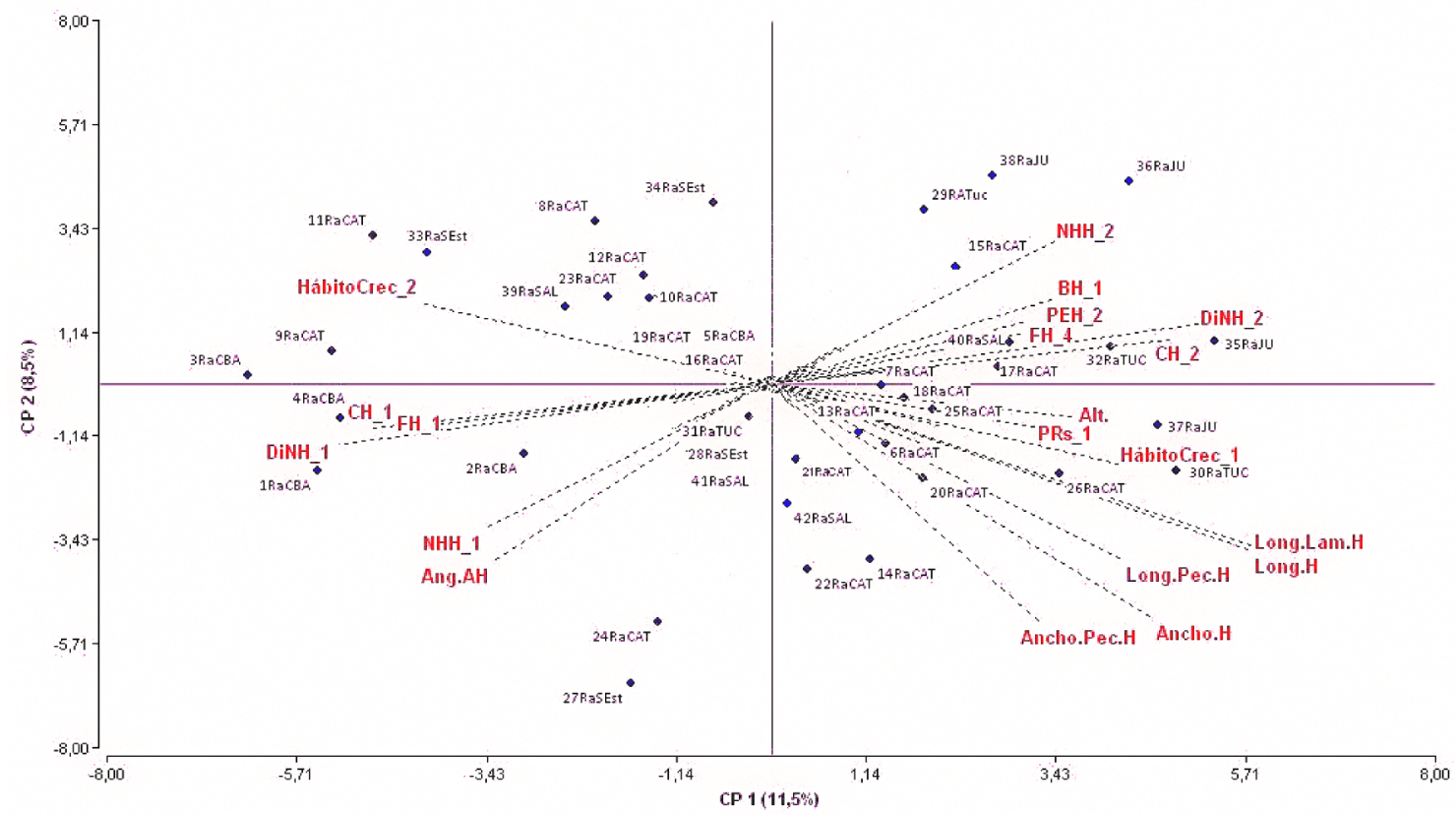

Fig. 3. Biplot CP1 vs. CP2. Proyección de los 42 individuos (puntos azules) y de las variables (en rojo) más vinculadas a estas $C P$, obtenida a partir del ACP en base a 25 variables morfológicas vegetativas (las mismas se pueden consultar en la Tabla 1a, Material Suplementario). 
(HábitoCrec_2) y hojas coriáceas (CH_1), ovadas (FH_1) y con nervaduras secundarias de ángulo de divergencia cercano a $90^{\circ}$ (DiNH_1).

En el dendrograma resultante del AC (Fig. 4), se pueden observar dos grupos formados a un nivel de corte de 26 utilizando la distancia Euclídea. En el Grupo 1 (en rojo en Fig. 4) se conglomeran 18 individuos: 15 de la región chaqueña y tres de la Selva Pedemontana. En el Grupo 2 (en azul en Fig. 4) se agrupan 24 individuos, la mayoría del sector pedemontano y de transición.
Como resultado de la prueba de KruskallWallis, se encontraron diferencias significativas entre conglomerados para todas las variables cuantitativas analizadas (Tabla 2, Material Suplementario). En el caso de las variables cualitativas, se encontraron diferencias significativas para: hábito de crecimiento, forma, base y consistencia de hoja, intensidad de resaltado de nervaduras en haz, ángulo de divergencia de nervaduras secundarias, densidad de nervaduras en hoja, grado de pilosidad de envés de hoja, de nervaduras en envés, de pecíolo

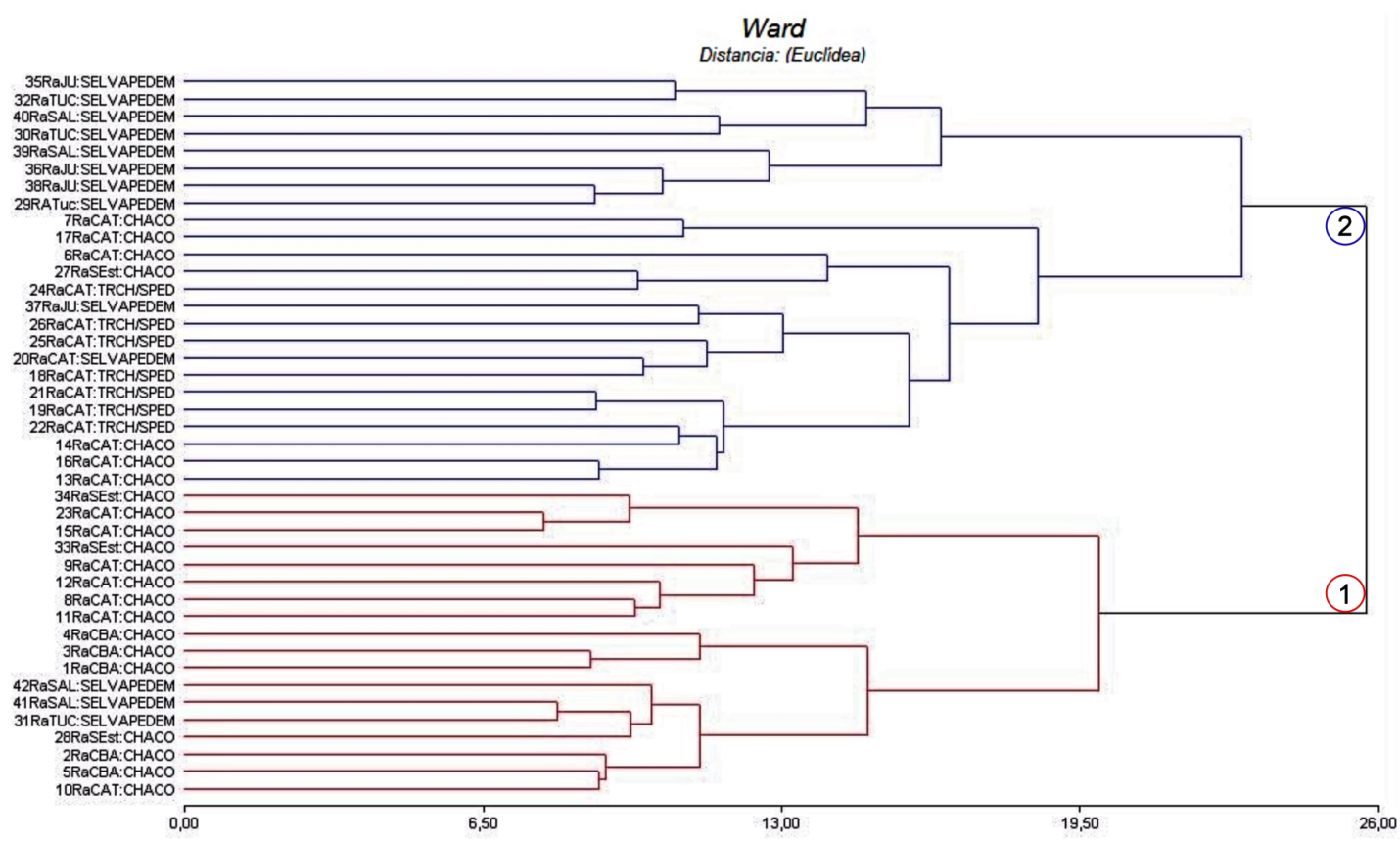

Fig. 4. Dendograma obtenido mediante el método de agrupamiento Ward y la distancia Euclídea, en base a 25 variables morfológicas vegetativas sobre 42 individuos. Las terminales de las ramas indican el código del ejemplar junto al tipo de ambiente correspondiente para cada uno. El Grupo 1 se indica en rojo y el Grupo 2 en azul.

Tabla 2. Valores de R (ANOSIM) y de F (PERMANOVA) entre los dos grupos morfológicos. Los valores de $p$ (entre corchetes) están corregidos según el criterio de Bonferroni.

\begin{tabular}{|ccc|}
\hline ANOSIM & GRUPO 1 & PERMANOVA \\
GRUPO 1 & & GRUPO 2 \\
GRUPO 2 & $0,1297[0,01]$ \\
\hline
\end{tabular}




\section{Á. Tessore et al. - Variabilidad morfológica de Ruprechtia apetala en Argentina}
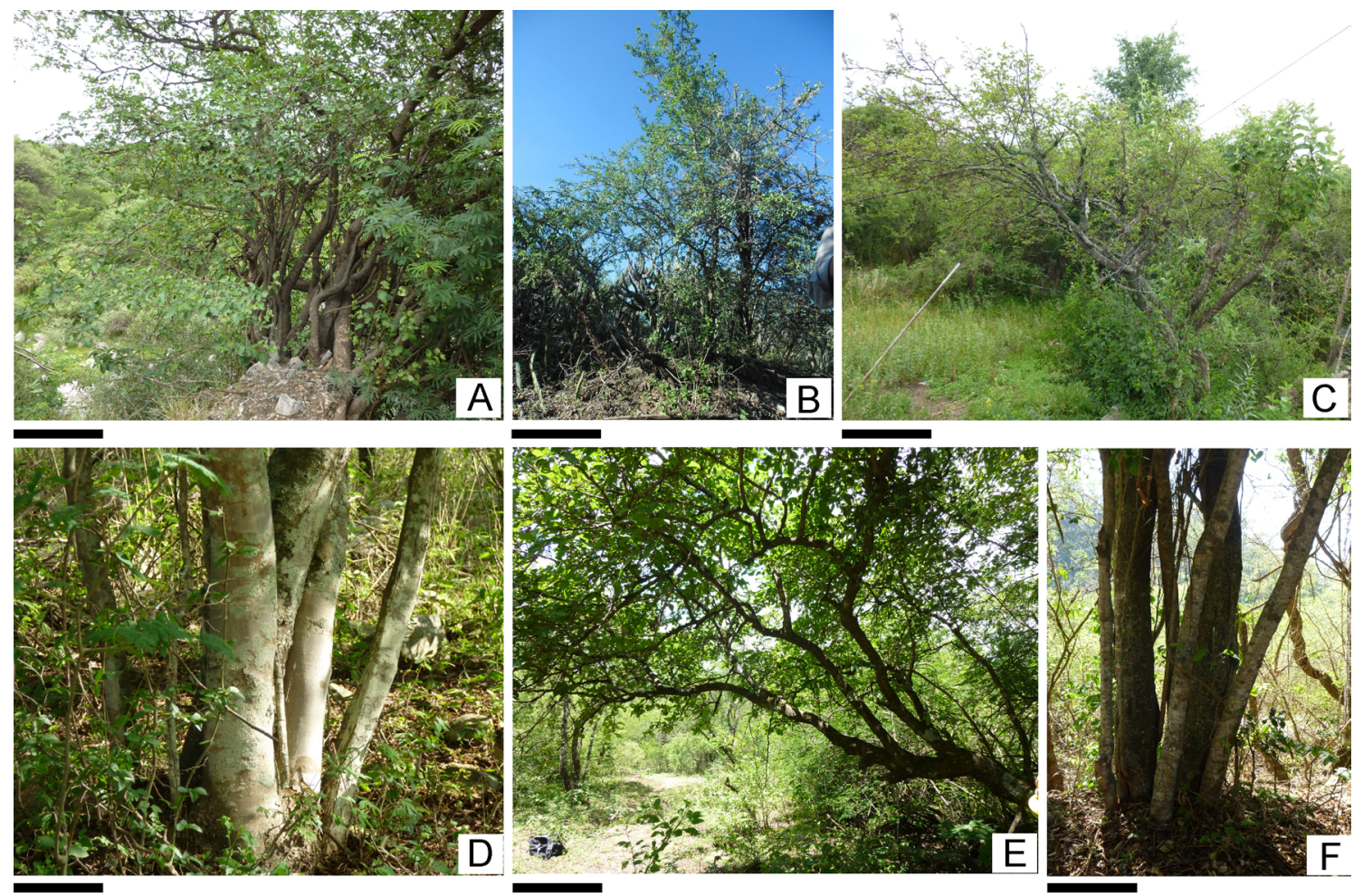

Fig. 5. A, B, C: Individuos de bajo porte, arbustivo, región Chaco Serrano (6RaCAT, 16RaCAT y 1RaCBA, respectivamente). D, E, F: Individuos de porte arbóreo, región Selva Pedemontana (38RaJU, 20RaTUC y 35RaJU, respectivamente). Escalas=A-B-C: $1 \mathrm{~m} ; \mathrm{D}: 10 \mathrm{~cm} ; \mathrm{E}: 1 \mathrm{~m} ; \mathrm{F}: 10 \mathrm{~cm}$.

y de ócrea. En las Fig. 5, 6 y 7 puede observarse parte de la variabilidad morfológica detectada.

A partir de los Análisis ANOSIM y PERMANOVA (Tabla 2), se evidencia que existe una diferencia global significativa entre ambos grupos (p-valor $<0,05$ ). No obstante, los valores de R y F (0,1297 y 2,95 , respectivamente) indican que existe una elevada similitud entre el Grupo 1 y el Grupo 2.

A partir de las variables con mayor importancia estadística en la caracterización de cada conglomerado (Tabla 3 ), se puede observar que los individuos del Grupo 1 se caracterizan por presentar nervaduras secundarias de ángulo de divergencia cercano a $90^{\circ}$ (DiNH_1), hojas coriáceas (CH_1), ovadas (FH_1), con bases obtusas a redondeadas (BH_7), con escasa pilosidad en el envés de la hoja (PEH_1) y hábito de crecimiento arbustivo (HábCrec_2). En relación a las variables cuantitativas, estos individuos presentan menores dimensiones en comparación al Grupo 2 para: altura (Alt), longitud de hoja, de lámina y de pecíolo (LongH, LongLamH, LongPecH) y ancho de hoja (AnchoH). Los individuos del Grupo 2 presentan frecuentemente nervaduras secundarias de ángulo de divergencia agudo (DiNH_2), hojas de consistencia membranácea $\left(\mathrm{CH}_{-} 2\right)$, elípticolanceoladas ( $\left.\mathrm{FH} \_4\right)$, de base agudas ( $\left.\mathrm{BH} \_1\right)$, y de hábito de crecimiento arbóreo (HábCrec_1). Para las variables cuantitativas se observaron mayores dimensiones en comparación al Grupo 1.

Por otra parte, las variables reproductivas, no incluidas en los análisis estadísticos, fueron incorporadas en una tabla realizada con fines descriptivos (Tabla 3, Material suplementario). En este sentido, se observó que el Grupo 1 presenta, usualmente, dimensiones más pequeñas que el Grupo 2 para las variables cuantitativas referidas a aquenio (LongAQ, AnchoAQ, LongPAQ, AngintLAQ, AngextLAQ). En cuanto a las variables 

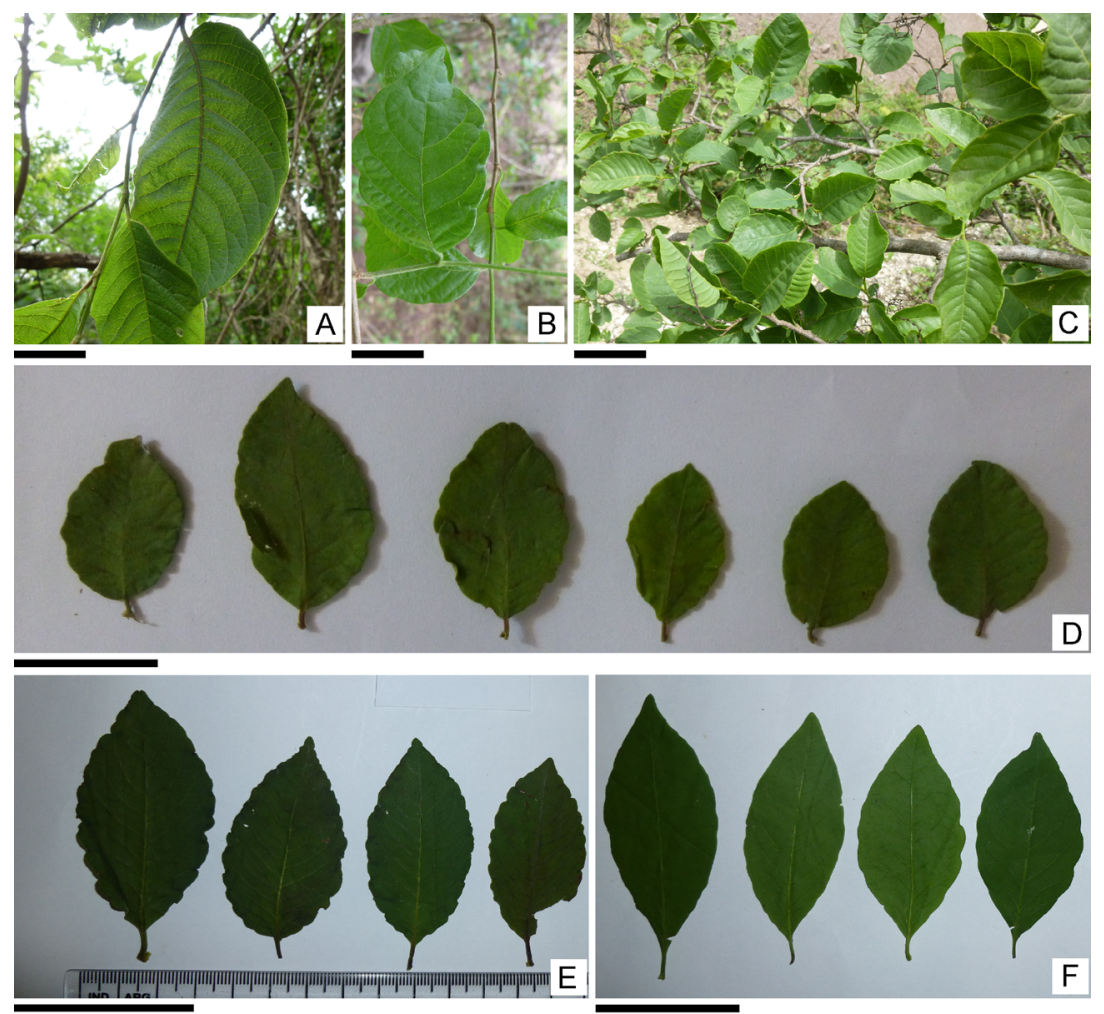

Fig. 6. Hojas, variabilidad de consistencia, formas y tamaños. A-B: hojas membranáceas (30RaTUC y 32RaTUC). C: hojas coriáceas (22RaCAT). D: principalmente ovadas a elíptico-ovadas (1RaCBA). E: elípticolanceoladas (7RaCAT). F: principalmente elípticas (36RaJU). Escalas= A-B: $1 \mathrm{~cm} ; \mathrm{C}: 5 \mathrm{~cm}$; D-E-F: $1 \mathrm{~cm}$.
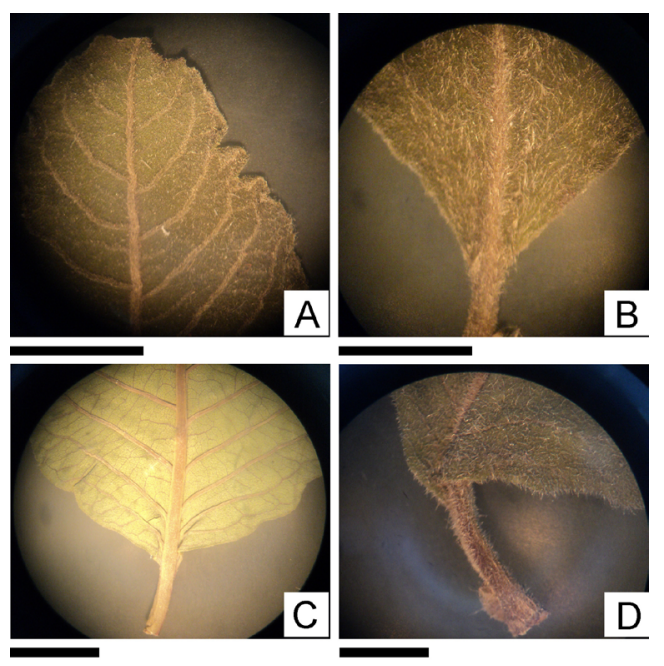

Fig. 7. Variabilidad de patrones de pilosidad. A-B: Pilosidad alta, en lámina y nervaduras del envés (A), y en haz (B). C: Ausencia de pilosidad en lámina y nervaduras del envés. D: pecíolo con abundante pilosidad. Escalas= A-B-C-D: $1 \mathrm{~cm}$. cualitativas, el Grupo 1 presenta una mayor frecuencia de forma de ápice de sépalo fructífero agudo a redondeado (FASF_1 y FASF_3), sépalo fructífero medianamente piloso (PSF_1), de color rosa pálido (CSF_6) y pedicelo medianamente piloso (PP_2). El Grupo 2, en cambio, demuestra mayor frecuencia en las variables forma de ápice de sépalo fructífero redondeado (FASF 3 ), sépalo fructífero medianamente piloso (PSF_1), de color naranja pálido (CSF_5) y pedicelo muy piloso (PP_3) (Tabla 3, Material suplementario). En la Fig. 8 se muestran imágenes representativas de la variabilidad de las estructuras reproductivas.

Desde el punto de vista biogeográfico, se observa una tendencia posicional de los individuos del Grupo 1 hacia la región chaqueña serrana, con pocos individuos en la región de Selva Pedemontana. Por su parte, los individuos del Grupo 2 se encuentran en la región pedemontana y transicional, con intrusiones hacia el sector chaqueño (ver mapa de la Fig. 9). 


\section{Á. Tessore et al. - Variabilidad morfológica de Ruprechtia apetala en Argentina}
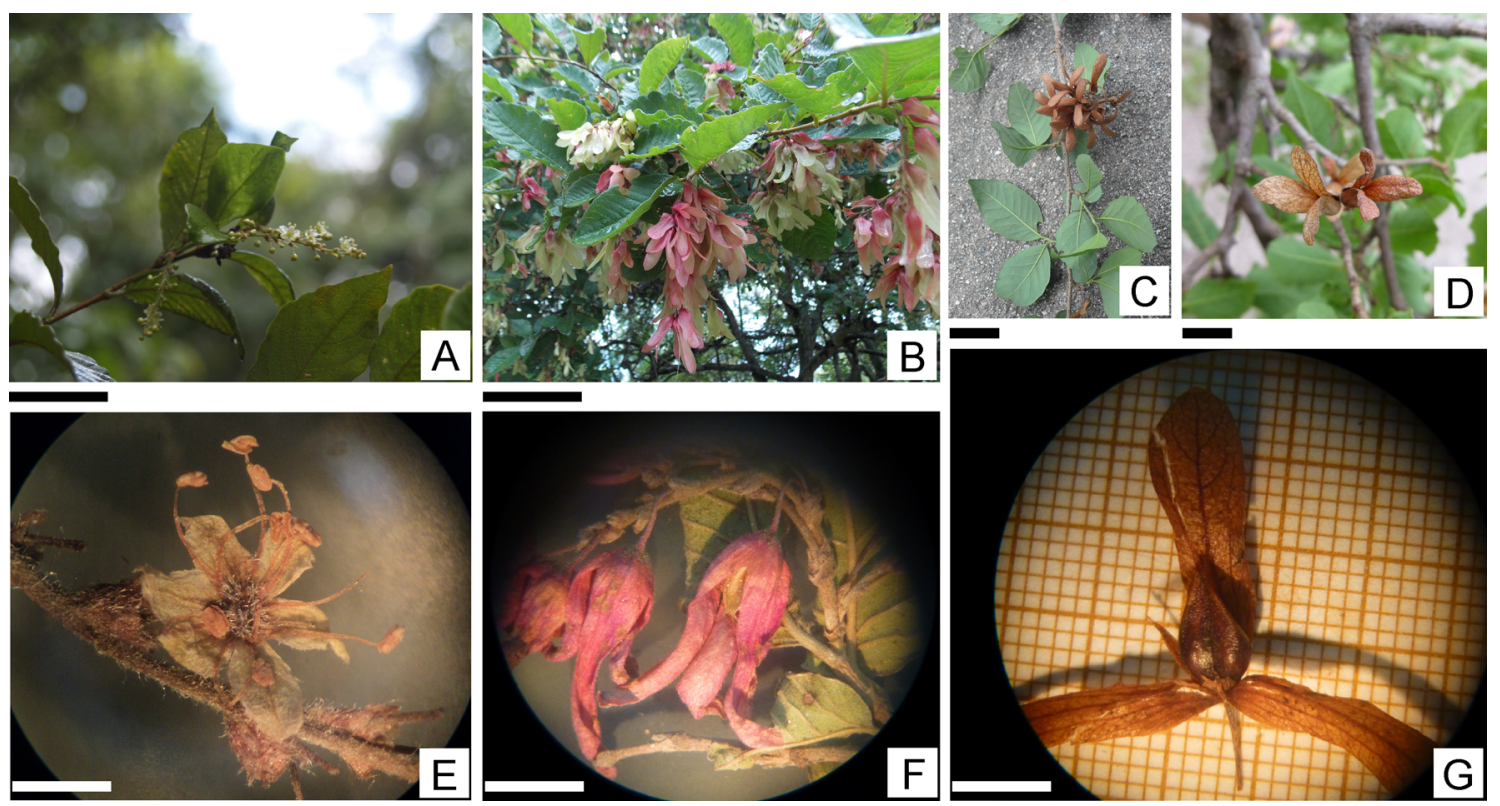

Fig. 8. Aspectos reproductivos de $R$. apetala. A-B: inflorescencias racemosas, masculina (A) y femenina (B). C-D: aquenios sobre ramas. E-F: vista en lupa de flor masculina $(E)$ y femenina $(F)$. G: aquenio, detalle en lupa. Escalas A-B= $1 \mathrm{~cm}$; C: $5 \mathrm{~cm}$; D: $1 \mathrm{~cm}$; E-F: 0,5 cm; G: $1 \mathrm{~cm}$.

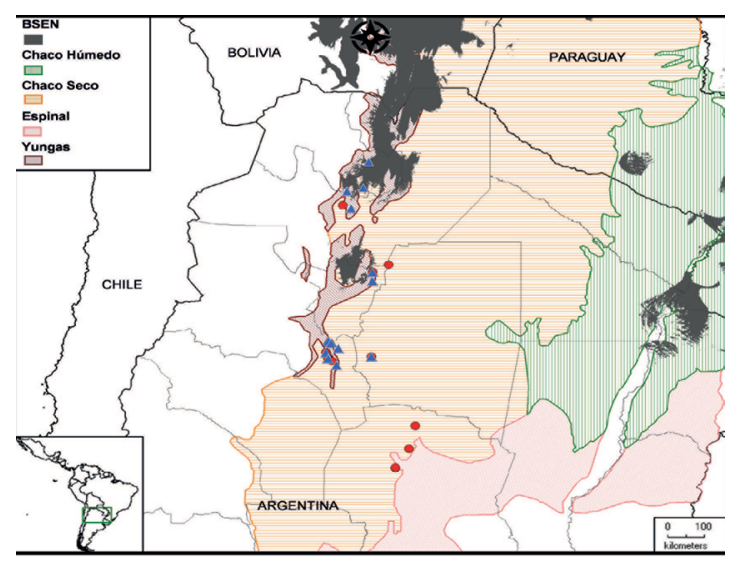

Fig. 9. Mapa de distribución de individuos colectados, Grupo 1 y Grupo 2 representados con círculos y triángulos, respectivamente. Se indican con distintos motivos las regiones biogeográficas abarcadas en el presente estudio, y otras regiones vecinas: Chaco Seco, Chaco Húmedo, Espinal, Yungas Andinas del Sur (Olson et al., 2001) y los Bosques Secos Estacionales Neotropicales (BSEN) (Särkinen et al., 2011).

\section{Discusión y Conclusiones}

En el presente estudio se encontraron caracteres vegetativos que, aunque no exclusivos, permiten distinguir dos grupos. El primer grupo está mayormente conformado por individuos colectados en la región chaqueña serrana, de menor porte (de hasta $6 \mathrm{~m}$ de alto) y diámetro (promedio de $7 \mathrm{~cm}$ ), con hojas de dimensiones más pequeñas, generalmente coriáceas, elípticas a redondeadas, con base obtusa a redondeada y nervaduras que mantienen un ángulo de divergencia cercano a $90^{\circ}$ con respecto a la vena central (ver Tabla 3 ). El segundo grupo se caracteriza por presentar individuos mayormente colectados en la Selva Pedemontana, frecuentemente de hábito arbóreo (o pequeños arbolitos) (de hasta $12 \mathrm{~m}$ de alto y con un diámetro promedio de $13 \mathrm{~cm}$ ), con hojas de mayores dimensiones, generalmente membranáceas, elíptico-lanceoladas, con base aguda y nervaduras secundarias con ángulo de divergencia agudo (ver Tabla 3 ).

No obstante, se evidencia una amplia variabilidad para un gran número de caracteres, tanto entre 


\section{Tabla 3. Variables vegetativas significativas para el Grupo 1 y Grupo 2, ordenadas según los valores} crecientes de $\mathrm{p}$. Valores de variables cualitativas refieren a frecuencias medias entre 0 y 1.

\begin{tabular}{|c|c|c|c|c|c|c|}
\hline \multirow[b]{2}{*}{ Variables Vegetativas } & \multirow[b]{2}{*}{ Abreviaturas } & \multicolumn{2}{|c|}{ GRUPO 1} & \multicolumn{2}{|c|}{ GRUPO 2} & \multirow[b]{2}{*}{ P-Valor } \\
\hline & & $\begin{array}{l}\text { Media/ } \\
\text { Frecuencia }\end{array}$ & $\begin{array}{l}\text { Desvío } \\
\text { Estándar }\end{array}$ & $\begin{array}{l}\text { Media/ } \\
\text { Frecuencia }\end{array}$ & $\begin{array}{l}\text { Desvío } \\
\text { Estándar }\end{array}$ & \\
\hline $\begin{array}{l}\text { Ángulo de divergencia de nervaduras } \\
\text { secundarias (cercano a } 90^{\circ} \text {-abiertas) }\end{array}$ & DiNH_1 & 0.73 & - & 0.34 & - & 0.000 \\
\hline $\begin{array}{l}\text { Ángulo de divergencia de nervaduras } \\
\text { secundarias (agudo-cerradas) }\end{array}$ & DiNH_2 & 0.27 & - & 0.66 & - & 0.000 \\
\hline Consistencia (coriácea) & CH_1 & 0.78 & - & 0.21 & - & 0.000 \\
\hline Longitud de lámina (cm) & LongLamH & 3.92 & 1.46 & 5.57 & 1.65 & 0.000 \\
\hline Consistencia (membranácea) & $\mathrm{CH} \_2$ & 0.22 & - & 0.79 & - & 0.000 \\
\hline Longitud de hoja (cm) & LongH & 4.28 & 1.54 & 6.01 & 1.74 & 0.000 \\
\hline Grado de pilosidad de envés (escasa) & PEH_1 & 0.83 & - & 0.49 & - & 0.001 \\
\hline Ancho de hoja (cm) & AnchoH & 2.34 & 0.86 & 3.08 & 0.87 & 0.001 \\
\hline Longitud de pecíolo (cm) & LongPecH & 0.40 & 0.16 & 0.51 & 0.18 & 0.002 \\
\hline Forma de hoja (elíptico-lanceoladas) & $\mathrm{FH} \_4$ & 0.04 & - & 0.16 & - & 0.002 \\
\hline Altura individuo $(\mathrm{m})$ & Alt & 3.12 & 1.75 & 5.81 & 3.01 & 0.003 \\
\hline Base de hoja (aguda) & $\mathrm{BH} \_1$ & 0.06 & - & 0.24 & - & 0.004 \\
\hline Forma de hoja (aovada) & $\mathrm{FH} \_1$ & 0.22 & - & 0.10 & - & 0.009 \\
\hline Ancho de pecíolo (cm) & AnchoPecH & 0.10 & 0.03 & 0.11 & 0.04 & 0.009 \\
\hline $\begin{array}{l}\text { Abundancia de pilosidad de } \\
\text { nervaduras en envés (media) }\end{array}$ & AbPNEH_3 & 0.57 & - & 0.31 & - & 0.013 \\
\hline Hábito de crecimiento (arbusto) & HábCrec_2 & 0.33 & - & 0.04 & - & 0.013 \\
\hline Hábito de crecimiento (árbol) & HábCrec_1 & 0.67 & - & 0.96 & - & 0.013 \\
\hline Base de hoja (obtusa-redondeada) & BH_7 & 0.22 & - & 0.11 & - & 0.030 \\
\hline Forma de hoja (lanceolada-oblonga) & FH_8 & 0.04 & - & 0.02 & - & 0.046 \\
\hline
\end{tabular}

como dentro de los grupos aquí definidos. Es por ello que no se encontró ninguna variable cualitativa para la cual alguno de los dos grupos haya presentado una frecuencia media absoluta cercana a 1 ó 0 , ni tampoco se observaron rangos excluyentes en el caso de las variables cuantitativas. Los individuos que presentaron mayor variabilidad morfológica provienen de zonas transicionales entre la Selva Pedemontana y el Chaco Serrano, donde se concentró buena parte de las colectas. Es posible que este ambiente transicional, con amplios rangos de variabilidad ambiental, se vea reflejado en la variabilidad morfológica observada.

Varios autores han estudiado la relación entre determinadas morfologías foliares y tipos particulares de ambiente, es decir, el grado de 


\section{Á. Tessore et al. - Variabilidad morfológica de Ruprechtia apetala en Argentina}

asociación del patrón morfológico que presenta una especie frente a determinadas condiciones ambientales a las que se encuentra expuesta (Landrum, 1986; Fonseca et al., 2000; McDonald et al., 2003; Warwick \& Lewis, 2009; Bünger et al., 2016; Prieto et al., 2016). En el caso estudiado, la región de la Selva Pedemontana, donde se encontraron mayoritariamente los individuos descriptos para el segundo grupo, se caracteriza por presentar suelos más fértiles y precipitaciones más abundantes (Prado, 1995, 2000; Mogni et al., 2015), con lo cual es esperable encontrar poblaciones con individuos que alcanzan mayores tamaños y que presentan un determinado tipo de morfología foliar más adaptado a un ambiente mesofítico (McDonald et al., 2003). En cambio, el sector centro-oeste del país donde se extiende el Chaco Serrano, caracterizado por sus suelos pedregosos y clima más seco, de menores precipitaciones (Ragonese \& Castiglioni, 1970; Cabrera, 1971; Cabrera \& Willink, 1973), se corresponde con un mayor predominio de aquellos individuos que presentaron el tipo de morfología descripto para el primer grupo, propio de un ambiente xerofítico. Efectivamente, se ha establecido que en los bosques del Distrito Chaqueño Serrano las especies características son predominantemente de hojas pequeñas con consistencia desde carnosas hasta subcoriáceas y coriáceas, como así también glabras o con baja densidad de tricomas (Arambarri et al., 2011).

De esta manera, se observa que los fenotipos descriptos para cada grupo se ven influidos por las condiciones ambientales de la región geográfica de procedencia. Sin embargo, ambos grupos no se encuentran restringidos a un único ámbito biogeográfico, sino que presentan un claro solapamiento en sus distribuciones. Por lo tanto, se podría decir que la especie en cuestión agrupa individuos que presentan una variabilidad morfológica notable a campo, que en cada caso parece corresponderse con un determinado tipo de ambiente, pero no se encontró ningún carácter que haya demostrado exclusividad para uno u otro grupo.

Las distintas poblaciones de una misma especie pueden diferenciarse fisiológica y morfológicamente por factores bióticos, edáficos o microclimáticos, como así también a lo largo del gradiente climático en el que ésta se extiende, dando lugar a "ecotipos" (Daubenmire, 1982). Estos pueden formarse como una respuesta de plasticidad adaptativa de los individuos de una población que ocupan ambientes heterogéneos (Pedroso et al., 2010). Dado que en este estudio se colectaron ejemplares de $R$. apetala a lo largo de gran parte de su rango de distribución en Argentina, y que tales ejemplares mostraron diferencias morfológicas según su procedencia, se podría decir que esa diversidad de ambientes sería la principal causa de la variabilidad observada. En consecuencia, se puede postular la existencia de "ecotipos" en $R$. apetala. Para corroborar y profundizar el estudio de la ocurrencia de ecotipos, sería necesario cultivar y realizar análisis morfológicos y genéticos a partir de semillas procedentes de diferentes poblaciones encontradas en los distintos tipos de ambiente, lo cual excede ampliamente los objetivos del presente trabajo.

El amplio rango de variabilidad morfológica que presenta esta especie, aparentemente asociado a distintos ambientes, debe ser considerado en futuros estudios para su conservación como recurso genético. Además, es fundamental extender el muestreo hacia otras zonas donde se registra la presencia de $R$. apetala, tanto en Argentina como en Bolivia (v.g Dptos. Anta y Orán, en Salta), a fin de contar con una descripción más completa y estudiar en profundidad su variabilidad morfológica en relación a las regiones donde habita.

\section{Contribución DE LOS AUTORES}

La idea e hipótesis fueron planteadas por DEP. AT, LJO y DEP coleccionaron especímenes y relevaron datos a campo. AT, LJO y VYM consultaron herbarios y bases de datos on line. VYM diseñó la metodología de toma de datos morfológicos y análisis estadístico. AT y VYM realizaron los análisis morfológicos y estadísticos, interpretación de los resultados y preparación de mapas y figuras. Todos los autores han realizado conjuntamente y en partes iguales la interpretación y redacción del manuscrito. Los autores declaran no tener conflictos de interés.

\section{Agradecimientos}

A la Facultad de Ciencias Agrarias, Universidad Nacional de Rosario. A los herbarios CTES, LIL y UNR, por la autorización para examinar ejemplares. A Eugenia Pedrero, Jorgelina Asmus, 
Roberto Salinas y Exequiel Agüero, por su ayuda en las colectas. A los dos revisores anónimos que contribuyeron a mejorar el manuscrito original. Este trabajo fue parcialmente financiado por la Universidad Nacional de Rosario (Proyecto AGR-200). DEP agradece al CONICET (Consejo Nacional de Investigaciones Científicas y Técnicas).

\section{Biblografía}

ARAMBARRI, A. M., M. C. NOVOA, N. D. BAYON, M. P. HERNÁNDEZ, M. N. COLARES \& C. MONTI. 2011. Ecoanatomía foliar de árboles y arbustos de los distritos chaqueños occidental y serrano (Argentina). Bol. Soc. Argent. Bot. 46: 251270.

BÜNGER, M. D. O., P. EINSEHLOR, M. L. N. FIGUEIREDO\&J.R.STEHMANN. 2016. Resolving Species Delimitations in the Eugenia involucrata Group (Eugenia sect. Phyllocalyx - Myrtaceae) with Morphometric Analysis. Syst. Bot. 40: 995-1002. https://doi.org/10.1600/036364415X690030

CABIDO, M., M. L. CARRANZA, A. ACOSTA \& A. PÁEZ. 1991. Contribución al conocimiento fitosociólogico del Bosque Chaqueño Serrano en la provincia de Córdoba, Argentina. Phytocoenologia 19: 547-566. https://doi.org/10.1127/phyto/19/1991/547

CABRERA, A. L. 1971. Fitogeografía de la República Argentina. Bol. Soc. Argent. Bot. 14: 1-42.

CABRERA, A. L. \& A. WILLINK. 1973. Biogeografia de América Latina. OEA, Washington DC (USA).

CIALDELLA, A. M. \& J. BRANDBYGE. 2001. Polygonaceae. En SPICHIGER, R. \& L. RAMELLA (eds.), Flora del Paraguay 33, pp. 1-106. Conservatoire et Jardin botaniques de la Ville de Genève \& Missouri Botanical Garden.

COCUCCI, A. E. 1958. El género Ruprechtia (Polygonaceae) en Argentina, Paraguay y Uruguay. Trab. Mus. Bot. Córdoba 2: 559-618.

COCUCCI, A. E. 1961. Revisión del género Ruprechtia (Polygonaceae). Kurtziana 1: 217-269.

DAUBENMIRE, R. F. 1982. Ecología vegetal: Tratado de Autoecología de Plantas. Limusa, México.

DE LA PEÑA, M. R. \& J. F. PENSIERO. 2004. Plantas Argentinas. Catálogo de nombres comunes. LOLA, Buenos Aires.

DEMAIO, P., U. O. KARLIN \& M. MEDINA. 2002. Árboles nativos del centro de Argentina. LOLA, Buenos Aires.
DI RIENZO, J. A., F. CASANOVES, M. G. BALZARINI, L. GONZALEZ, M. TABLADA \& C. W. ROBLEDO. 2013. InfoStat versión 2013. Grupo InfoStat, FCA, Universidad Nacional de Córdoba, Argentina. Disponible en: http://www.infostat.com.ar

DIGILIO, A. P. \& P. R. LEGNAME. 1966. Los árboles indígenas de la provincia de Tucumán. Opera Lilloana 15: 1-107.

FONSECA, C. R., J. M. OVERTON, B. COLLINS \& M. WESTOBY. 2000. Shifts in trait-combinations along rainfall and phosphorus gradients. J. Ecol. 88: 964977.

https://doi.org/10.1046/j.1365-2745.2000.00506.x

GRISEBACH, A. H. R. 1874. Plantae Lorentzianae. Abh. Königl. Ges. Wiss. Göttingen. 19: 49-279.

HAMMER, Ø., D. HARPER \& P. D. RYAN. 2001. PAST: Paleontological statistics software package for education and data analysis. Palaeontol. Electron. 4: 9.

HERZOG, T. C. J. 1922. Herzog's Bolivianische Pflanzen, VI. Meded. Rijks-Herb. 46: 1-31.

HIJMANS, R., L. GUARINO, A. JARVIS \& R. O'BRIEN. 2007. DIVA-GIS v.7.3.0.1. Disponible en: http://www.diva-gis.org

INFOSTAT. 2004. Grupo INFOSTAT, FCA, Universidad Nacional de Córdoba, Argentina. Versión 2004. 314 p.

JSTOR GLOBAL PLANTS. 2000. ITHAKA [online]. Disponible en: http://www.plants.jstor.org/ [Acceso: Agosto 2019].

LANDRUM, L.R .1986. Campomanesia, Pimenta, Blepharocalyx, Legrandia, Acca, Myrrhinium and Luma. Fl. Neotrop. Monogr. 45: 1-178.

LÊ, S., J. JOSSE \& F. HUSSON. 2008. FactoMineR: an R package for multivariate analysis. J. Stat. Softw. 25: 1-18. https://doi.org/10.18637/jss.v025.i01

MCDONALD, P. G., C. R. FONSECA, J. M. C. OVERTON \& M. WESTOBY. 2003. Leaf-size divergence along rainfall and soil-nutrient gradients: is the method of size reduction common among clades?. Funct. Ecol. 17: 50-57. https://doi.org/10.1046/j.1365-2435.2003.00698.x

MISSOURI BOTANICAL GARDEN. 2011. Tropicos. org [online]. Disponible en: http://www.tropicos.org/ [Acceso: Diciembre 2019].

MOGNI, V. Y., L. J. OAKLEY, H. M. MATURO, L. A. GALETTI \& D. E. PRADO. 2015. Biogeografia e florística dos Bosques Secos Estacionais Neotropicais (BSEN). OKARA-Geografia em debate 9: 275-296.

MORALES, J. M., M. SIROMBRA \& A. D. BROWN. 1995. Riqueza de árboles en las Yungas argentinas. En: BROWN A. \& H. GRAU (eds.). Investigación, 


\section{Á. Tessore et al. - Variabilidad morfológica de Ruprechtia apetala en Argentina}

Conservación y Desarrollo en Selvas Subtropicales de Montaña, pp. 163-174. L.I.E.Y. (UNT), Proyecto de Desarrollo Agroforestal, Tucumán, Argentina.

OLSON, D. M., E. DINERSTEIN, E. D. WIKRAMANAYAKE, N. D. BURGESS, G. V. POWELL, E. C. UNDERWOOD \& C. J. LOUCKS. 2001. Terrestrial Ecoregions of the World: A New Map of Life on Earth. BioScience 51: 933-938.

h t t p s://doi .org/10.1641/00063568(2001)051[0933:TEOTWA]2.0.CO;2

OYARZABAL, M., J. CLAVIJO, L. OAKLEY, F. BIGANZOLI, P. TOGNETTI, I. BARBERIS, H. M. MATURO, R. ARAGÓN, P. I. CAMPANELLO, D. PRADO, M. OESTERHELD \& R. J. C. LEÓN. 2018. Unidades de vegetación de la Argentina. Ecol. Austral 28: 40-63. https://doi.org/10.25260/EA.18.28.1.0.399

PEDROSO, H. L., L. C. D. ROCHA-FILHO \& C. LOMÔNACO. 2010. Variación fenotípica de plantas del Cerrado (Sabana brasileña) frente a la heterogeneidad ambiental. Ecosistemas 19: 24-36.

PENDRY, C. A. 2004. Monograph of Ruprechtia (Polygonaceae). Syst. Bot. Monogr. 67: 1-113. https://doi.org/10.2307/25027911

PRADO, D. E. 1995. Selva pedemontana: contexto regional y lista florística de un ecosistema en peligro. En: BROWN A. \& H. GRAU (eds.). Investigación, Conservación y Desarrollo en Selvas Subtropicales de Montaña, pp. 19-52. L.I.E.Y. (UNT), Proyecto de Desarrollo Agroforestal, Tucumán, Argentina.

PRADO, D. E. 2000. Seasonally dry forests of tropical South America: from forgotten ecosystems to a new phytogeographic unit. Edinburgh J. Bot. 57: 437461. https://doi.org/10.1017/S096042860000041X
PRIETO, A., V. N. ISPIZÚA \& A. M. CLAUSEN. 2016. Distribución y variabilidad morfológica de poblaciones de Solanum commersonii (Solanaceae) en la región pampeana de la Argentina. Bol. Soc. Argent. Bot. 51: 59-71.

R CORE TEAM. 2015. R: A language and environment for statistical computing. R Foundation for Statistical Computing, Vienna, Austria. Disponible en: http:// www.R-project.org.

RAGONESE, A. \& J. C. CASTIGLIONI. 1970. La vegetación del Parque Chaqueño. Bol. Soc. Argent. Bot. 11: 133-160.

ROHLF, F. 2013. tpsDig2. 2.17 ed. SUNY, Stony Brook, NY.

SÄRKINEN，T., J. R. IGANCI，R. LINARESPALOMINO, M. F. SIMON \& D. E. PRADO. 2011. Forgotten forests-issues and prospects in biome mapping using Seasonally Dry Tropical Forests as a case study. B. M. C. Ecol. 11: 1.

TURLAND, N., J. WIERSEMA, F. BARRIE, W. GREUTER, D. HAWKSWORTH, P. HERENDEEN, S. KNAPP, W. H. KUSBER, D. Z. LI, K. MARHOLD, T. MAY, J. MCNEILL, A. MONRO, J. PRADO, M. PRICE \& G. SMITH (eds.). 2018. International Code of Nomenclature for algae, fungi, and plants (Shenzhen Code) adopted by the Nineteenth International Botanical Congress Shenzhen, China, July 2017. Reg. Veg. 159. Koeltz Botanical Books, Glashütten. https://doi.org/10.12705/Code.2018

WARWICK, M. C. \& G. P. LEWIS. 2009. A revision of Cenostigma (Leguminosae-CaesalpinioideaeCaesalpinieae), a genus endemic to Brazil. Kew Bull. 64: 135-146. https://doi.org/10.1007/s12225-008-9091-1

WEDDELL, H. A. 1849. Additions a la Flore de L'Amérique du Sud. Ann. Sci. Nat. Bot. 3: 249-268. 
Bol. Soc. Argent. Bot. 55 (3) 2020

\title{
Material Suplementario
}

\author{
1a- Especímenes colectados
}

Los ejemplares citados a continuación, corresponden a colectas de Tessore et al., depositados en UNR.

\begin{abstract}
ARGENTINA. Catamarca: Dpto. El Alto. Entre El Lindero e Infanzón, -28,5849 S -65,516 O, 1212 msnm, 24/11/2014, 24RaCAT, 25RaCAT, 26RaCAT. Dpto. Fray Mamerto Esquiú. Ingreso a Villa Las Pirquitas, -28,2874 S -65,727 O, 679 msnm, 22/11/2014, 6RaCAT. -28,2874 S -65,727 O, 679 msnm, 22/11/2014, 7RaCAT. Villa Las Pirquitas, a $20 \mathrm{~km}$ de la capital, -28,2755 S -65,733 O, $713 \mathrm{msnm}, 22 / 11 / 2014,8 \mathrm{RaCAT}$. Villa Las Pirquitas, -28,2753 S -65,7332 O, 712 msnm, 22/11/2014, 9RaCAT. -28,2742 S -65,7335 O, 716 msnm, 22/11/2014, 10RaCAT. -28,2742 S -65,7335 O, 716 msnm, 22/11/2014, 11RaCAT. -28,2742 S 65,7335 O, 716 msnm, 22/11/2014, 12RaCAT. Villa Las Pirquitas (cancha de Las Pirquitas), -28,2805 S-65,7331 O, 688 msnm, 22/11/2014, 13RaCAT. -28,2805 S -65,7331 O, $688 \mathrm{msnm}, 22 / 11 / 2014,14 \mathrm{RaCAT}$. San Antonio, Refugio de vida silvestre Merced de Alpatauca, -28,4171 S -65,6901 O, 565 msnm, 22/11/2014, 15RaCAT. -28,4199 S -65,6884 O, 575 msnm, 22/11/2014, 16RaCAT. -28,4178 S -65,6863 O, 574 msnm, 22/11/2014, 17RaCAT. Dpto. Paclin. Cerro San Antonio de Paclín, -28,0166 S -65,6985 O, 1106 msnm, 23/11/2014, 18RaCAT. -28,0166 S -65,6985 O, 1106 msnm, 23/11/2014, 19RaCAT. Ruta 38 que va a Tucumán, Cuesta del Totoral, -28,0564 S -65,6108 O, 701 msnm, 23/11/2014, 20RaCAT. Dpto. Santa Rosa. Pie de Cuesta de Alijilán a El Alto, -28,1896 S -65,4722 O, 608 msnm, 23/11/2014, 21RaCAT. -28,1896 S -65,4722 O, 608 msnm, 23/11/2014, 22RaCAT. Dpto. Valle viejo. Cuesta del Portezuelo, -28,4711 S -65,6183 O, $948 \mathrm{msnm}, 24 / 11 / 2014$, 23RaCAT. Córdoba: Dpto. Colón. Agua de Oro, Parque Tres Cóndores, Cód. 5107, Calle El Quebracho, -31,0149 S -64,3039 O, 832 msnm, 20/11/2014, 1RaCBA. -31,0144 S -64,3034 O, 829 msnm, 20/11/2014, 2RaCBA. Dpto. Sobremonte. Camino a Cerro Colorado, -30,0161 S -63,8928 O, 506 msnm, 20/11/2014, 4RaCBA, 5RaCBA. Dpto. Totoral. Oeste de Las Peñas, -30,5591 S -64,0213 O, 593 msnm, 20/11/2014, 3RaCBA. - Jujuy: Dpto. El Carmen. Camino de Cornisa, ruta 9 (pasando el paraje Los Naranjos), -24,4548 S -65,2956 O, 1256 msnm, 29/11/2014, 38RaJU. Dpto. Ledesma. Parque Nacional Calilegua (zona de camping), -23,7621 S -64,8511 O, $621 \mathrm{msnm}, 29 / 11 / 2014$, 35RaJU, 36RaJU. Dpto. San Pedro. Ruta 34 cerca de Barro Negro, -24,3617 S -64,966 O, 865 msnm, 29/11/2014, 37RaJU. - Salta: Dpto. Capital. Monolito a Güemes, Cañada de La Horqueta, -24,867 S -65,21 O, 1150 msnm, 30/11/2014, 39RaSAL, 40RaSAL. Cerro San Bernardo, -24,7891 S -65,3924 O, 1468 msnm, 30/11/2014, 41RaSAL. -24,7895 S -65,393 O, 1463 msnm, 30/11/2014, 42RaSAL. - Santiago del Estero: Dpto. Choya. Villa La Punta, Sierra de Guasayán (parte baja), -28,372 S -64,8006 O, 425 msnm, 25/11/2014, 27RaSEst, 28RaSEst. Dpto. Pellegrini. Cerro El Remate, -26,1992 S -64,4461 O, 397 msnm, 27/11/2014, 33RaSEst. -26,1982 S -64,4464 O, 417 msnm, 27/11/2014, 34RaSEst. - Tucumán: Dpto. Burruyacu. Aguas Blancas, -26,3695 S -64,7784 O, 732 msnm, 26/11/2014, 29RaTUC, 30RaTUC, 31RaTUC. El Puestito (de arriba), -26,5857 S -64,7759 O, 723 msnm, 26/11/2014, 32RaTUC.
\end{abstract}

\section{1b-Material adicional examinado}

ARGENTINA. Catamarca: Dpto. Capayán. Ruta 38, km 538, entre Capayán y Huillapima, -28,75 S -66,01 O, 446 msnm, 25/02/2008, Pensiero et al. 7484 (CTES). - Córdoba: Dpto. Colón. En las estribaciones de la sierra de Ascochinga, 01/04/1871, Lorentz 372 (holotipo de R. corylifolia: GOET). En un radio de Caleras de las sierras de Córdoba, 30/04/1877, Hieronymus s.n. (K). Dpto. Cruz del Eje. Sierra de Serrezuela: entre Tuclame y Serrezuela, 14/04/1951, Hunziker 9110 (CTES). Dpto. Punilla. La Falda, 05/04/1983, Pire 1228 (CTES). Mallín, 900 msnm, 19/02/1951, Gutiérrez 282 (LIL, CTES). Camino de Capilla del Monte a San Marcos Sierra, 18/04/1947, Boelcke y Serrano 2662 (CTES). San Marcos Sierra, 24/01/1951, de la Sota 4104 (CTES). Dpto. San Javier. 9 km al sur de La Paz, -32,29 S -65,03 O, 900 msnm, 15/01/2004, Pozner y Belgrano 238 (CTES). Dpto. Santa María. Comuna La Paisanita, 05/01/2007, Keller 4654 (CTES). Dpto. Sobremonte. A 30 km de San Francisco del Chañar, 20/01/1984, Renolfi 406 (CTES). - Jujuy: Dpto. El Carmen. Pampa Blanca, 16/11/1970, Cabrera y Fabris 20963 (CTES). Dique La Ciénaga, 06/01/1971, Krapovickas y Cristóbal 17539 (CTES). Las Trampas. 2 km al SE de Represa hidroeléctrica dique Las Maderas, 800-900 msnm, 25/02/1997 Protomastro 1147 (CTES). Campo experimental de la Facultad de Cs Agrarias UNJu, 02/1994, Ahumada 7125 (CTES). Dpto. San Pedro. Ruta prov. 56, $10 \mathrm{~km}$ de La Mendieta, camino a San Salvador de Jujuy, -24,33 S -65,03 O, 990 msnm, 19/02/1998, Morrone et al. 2796 (CTES). Entre San Pedro y 


\section{Á. Tessore et al. - Variabilidad morfológica de Ruprechtia apetala en Argentina}

Pampa Blanca, ruta 34, 02/02/1975, Zuloaga y Deginani 402 (CTES). Sierra de Zapla, 07/03/1940, Burkart y Troncoso 12019 (CTES). San Juan de Dios, 750 msnm, 16/03/1967, Fabris y Crisoi 7002 (CTES). Dpto. Santa Bárbara. Ruta de Santa Clara a Abra de Los Morteros, 3 km de Santa Clara, -24,3 S -64,63 O, 750 msnm, 28/02/1997, Zuloaga et al. 6357 (CTES). Ruta de Siete Aguas a Palma Sola, a $10 \mathrm{~km}$ de Palma Sola, -23,9 S -64,31 O, 600 msnm, 26/02/1997 Zuloaga et al. 6262 (CTES). Sierras del Maíz Gordo, 4 km del Infiernillo, camino al Puesto del Maíz Gordo, próximo al Río Maíz Gordo, -24,26 S -64,25 O, 1050 msnm, 22/02/1998, Morrone et al. 3030 (CTES). Sauce Guacho, 02/03/1983, Ahumada 4504 (CTES). - Salta: Dpto. Anta. Parque Nacional El Rey, sendero Los Ocultos, 11/03/2005, Ferraro y Popoff 7468 (CTES). Camino a Parque Nacional El Rey, $6 \mathrm{~km} \mathrm{~N}$ de la carretera, -24,83 S -64,66 O, 860 msnm, 25/09/1985, Gentry et al. 51810 (CTES). Dpto. Capital. Cerro al sur del San Bernardo y El Portezuelo, 500 $\mathrm{m}$ al SE del edificio de Radio Club Salta, 1250-1350 msnm, 26/01/1983, Novara 3077 (CTES). Cerro San Bernardo, 09/02/1936, Schinini 2850 (CTES). Sierras de Vélez, finca La Candelaria, $4-5 \mathrm{~km}$ E de la Universidad Católica de Salta, pasando Los Lapachos, $1300 \mathrm{msnm}, 22 / 02 / 2000$, Tolaba 2388 (CTES). $3 \mathrm{~km} \mathrm{~S}$ de ruta Nac. № 9, y el camino a Estancia La Cruz, 18/10/1994, Ahumada 7110 (CTES). 300 msnm, 16/02/1989, Cáceres 1898 (CTES). Dpto. Gral Güemes. Palomitas (4km al sur), 750 msnm, 04/01/1989, Saravia 1804 (CTES). Dpto. La Viña. Ruta 47, 6 km E de ruta 68, Chaco Húmedo, 25/01/2007, Paula-Souza et al. 7818 (CTES). Dpto. Metán. Ruta Nac 34, de Lumbrera a Río Juramento, -25,16 S -64,96 O, 13/02/1997, Zuloaga et al. 5818 (CTES). Dpto. Orán. Ruta prov. 18, a 21 km de Isla de Cañas, camino a Orán, 20/11/2001, Morrone et al. 3958 (CTES). Ruta 34, km 1290, $3 \mathrm{~km}$ al S del Arroyo Los Monos, $450 \mathrm{msnm}, 11 / 05 / 1989$, Novara et al. 8874 (CTES). Hipólito Irigoyen, interior de selva secundaria, 18/04/1998, Vanni et al. 4209 (CTES). Río Blanco, 30/03/1951, de la Sota 4500 (LIL, CTES). Dpto. Rosario de la Frontera. Los Baños a Balboa, 1947, O’Donell 5356 (LIL, CTES). Dpto. San Martín. 3 km E de Senda Hachada, río Seco, 02//04/1977, Krapovickas y Schinini 30816 (CTES). General Ballivián, prox. el Saladillo, 09/05/1974, Legano et al. s.n. (LIL, CTES). - Sgo. del Estero: Dpto. Choya. Ruta prov 34, alrededores de Villa La Punta, sobre el piedemonte de la Sierra de Guasayán, 21/01/2006, Biurrun y Callela 8107 (CTES). Dpto. Guasayán. Sierras de Guasayán, 25/09/1989, Perez et al. 4794 (CTES). Dpto. Ojo de Agua. Sierra de Ambargasta. Camino de Amimán a Lomitas Blancas, 01/12/1878, NN s.n. (CTES). - Tucumán: Dpto. La Cruz. En el subtrópico, Bosques de La Cruz, 20/04/1872, Lorentz 322 y 323 (sintipos de R. excelsa: GOET). Idem loc., 24/04/1872, Lorentz 324 (lectotipo de R. excelsa: GOET). Dpto. Trancas. Ruta 38, entre desvío a Cadillal y Tapia, 17/03/1971, Meyer s.n. (LIL, CTES).

BOLIVIA. Dpto. Chuquisaca: Prov. Luis Calvo. -20,66 S -63,16 O, 10/04/1993, Saravia Toledo et al. 11621 (CTES). Prov. Tomina. Matorral a lo largo de Río Pilcomayo, 01/12/1845, Weddell 3873. (lectotipo de R. apetala: P). - Dpto. La Paz: Prov. Franz Tamayo. -14,35 S -68,53 O, 886 msnm, 07/06/2005, Cayola 1726 (CTES). -14,47 S -68,53 O, 900 msnm, 23/02/2003, Canqui et al. 158 (CTES). -14,45 S -68 O, 900 msnm, 19/02/2002, Canqui et al. s.n. (CTES). -14,47 S -68,54 O, 951 msnm, 01/04/2014, Fuentes Claros 17812 y 17833 (LPB, MO). -14,32 S -68,57 O, 662 msnm, 01/04/2014, Fuentes Claros 18505, 18507, 18508 y 18516 (LPB, MO). -14,33 S -68,57 O, 1020 msnm, 08/04/2014, Fuentes Claros 18675, 18787, 18805, 18806 y18808 (LPB). Ídem loc., 830 msnm, 13/04/2014, Fuentes Claros 18925 (LPB). -14,54 S -68,69 O, 902 msnm, 24/04/2012, Quispe Paucara 83 (LPB). Prov. Inquisivi. Inquisivi, 1800 msnm, 01/12/1846, Weddell 4197 (lectotipo R. mollis: P). Ídem loc., 1500 msnm, 24/04/1992, Beck 21112 (LPB). Prov. Sud Yungas. 1200 msnm, 15/03/1999, Pendry et al. 624 (CTES). -16,66 S -67,51 O, 2000 msnm, 30/04/1995, Beck 22421 (CTES). - Dpto. Santa Cruz: Descenso desde Samaipata para Mairantal, 1700 msnm, 01/03/1911, Herzog 1781 (lectotipo de R. boliviensis: JE). Prov. Caballero. -18,09 S -64,52 O, $1630 \mathrm{msnm}$, 12/04/2006, Dematteis et al. 2374 (CTES). -18,51 S -64,55 O, 20/01/1995, Saravia 1487 (CTES). -18,06 S -64,4 O, 1575 msnm, 17/04/2002, Nee et al. 52181 (CTES). Prov. Cordillera. -18,66 S -63,25 O, 525 msnm, 24/03/2002, Nee et al. 51966 (CTES). -19,29 S -60,62 O, 300 msnm, 06/02/1998, Fuentes y Navarro 2164 (CTES). -18,45 S -63,2 O, 500 msnm, 14/03/1998, Nee 48678 (CTES). -18,28 S -63,2 O, 525 msnm, 27/02/1998, Nee 48502 (CTES). Prov. Florida, -18,18 S -64,2 O, 1750 msnm, 14/01/1998, Beck 23375 (CTES). -18,41 S -64,03 O, 1750 msnm, 14/01/1998, Beck s.n. (CTES). -18,02 S -64,1 O, 1225 msnm, 31/03/2002, Nee M, Sundue M y Carrasco A 52106 (CTES). -18,15 S -63,93 O, 1400 msnm, 08/03/1998 Nee et al. 48605 (CTES). Prov. Vallegrande. $1850 \mathrm{msnm}, 26 / 01 / 1994$, Saravia 2310-1 (CTES). - Dpto. Tarija: Prov. Arco. 1500 msnm, 05/02/1945, TM 17397 (LIL, CTES). Prov. Gran Chaco. -21,33 S -63,75 O, 04/05/1983 Krapovickas y Schinini 39172 (CTES). 
Tabla 1a. Caracteres vegetativos analizados sobre individuo, hoja, ócrea, rama y corteza. Aquellos considerados en los análisis estadísticos se encuentran resaltados en gris.

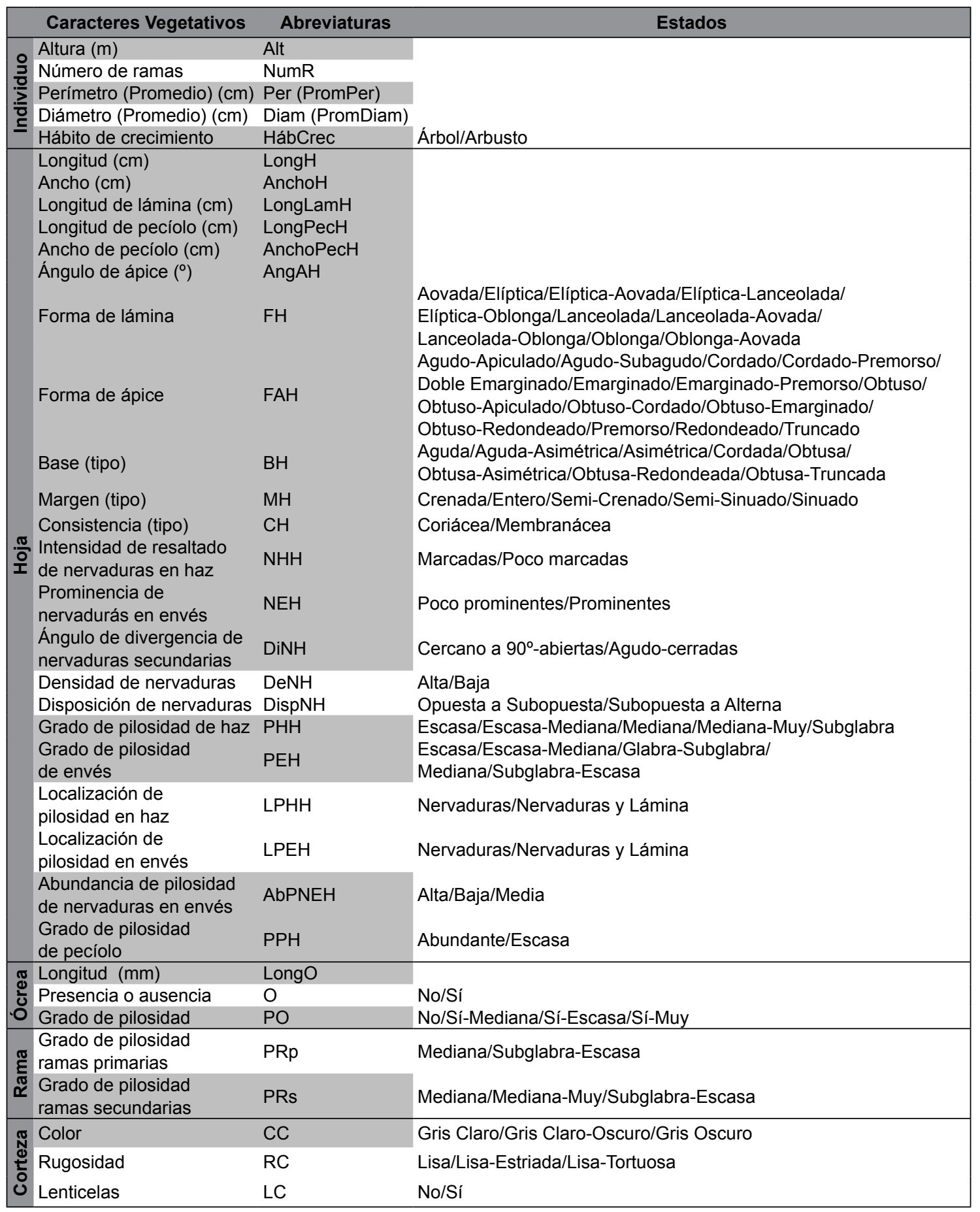




\section{Á. Tessore et al. - Variabilidad morfológica de Ruprechtia apetala en Argentina}

Tabla 1b. Caracteres reproductivos analizados sobre inflorescencia, flor y fruto.

\begin{tabular}{|c|c|c|c|}
\hline & Caracteres Reproductivos & Abreviaturas & Estados \\
\hline \multirow{7}{*}{ 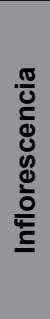 } & Longitud pedúnculo (mm) & LongPedl & \\
\hline & Longitud raquis (mm) & LongRaql & \\
\hline & Longitud brácteas (mm) & LongB1I & \\
\hline & Longitud bractéolas (mm) & LongB2I & \\
\hline & Ubicación & I & Axilar/Terminal \\
\hline & Presencia pilosidad & $\mathrm{PI}$ & No/Sí \\
\hline & Ubicación pilosidad & UPI & Brácteas-Bractéolas/Pedicelo/Pedúnculo/Raquis \\
\hline \multirow{10}{*}{ 흔 } & Pilosidad & PFL & No/Sí \\
\hline & Localización de pilosidad & LocPFL & Cáliz/Estilo/Estigma/Pedicelo \\
\hline & Coloración & CFL & Amarillo-verdosas/Rojizo-purpúreas \\
\hline & Sexo & SxFL & Femeninas/Masculinas \\
\hline & Longitud pedicelo (mm) & LongPedFL & \\
\hline & Longitud sépalos (mm) & LongSFL & \\
\hline & Ancho sépalos (mm) & AnchoSFL & \\
\hline & Longitud pétalos (mm) & LongPFL & \\
\hline & Ancho pétalos (mm) & AnchoPFL & \\
\hline & Longitud estilo/estigma (mm) & LongE/EFL & \\
\hline \multirow{15}{*}{$\stackrel{ }{\stackrel{2}{2}}$} & $\begin{array}{l}\text { Forma de apice de } \\
\text { sépalos fructíferos }\end{array}$ & FASF & Aguda/Obtusa/Redondeada/Retusa \\
\hline & $\begin{array}{l}\text { Grado de pilosidad de } \\
\text { sépalos fructíferos }\end{array}$ & PSF & Muy pilosos/Pilosos/Poco pilosos \\
\hline & Grado de pilosidad de pedicelo & PP & Glabro/Glabro-Piloso/Muy piloso/Piloso \\
\hline & Coloración de sépalos fructíferos & CSF & $\begin{array}{l}\text { Cobrizo/Marrón oscuro/Marrón pálido/Naranja } \\
\text { cobrizo/ Naranja páido/Rosa pálido }\end{array}$ \\
\hline & Longitud (mm) & LongAQ & \\
\hline & Ancho (mm) & AnchoAQ & \\
\hline & Longitud pedicelo (mm) & LongPAQ & \\
\hline & Largo lóbulo (mm) & LargoLAQ & \\
\hline & Ancho lóbulo (mm) & AnchoLAQ & \\
\hline & Ángulo interno lóbulo $\left({ }^{\circ}\right)$ & AngintLAQ & \\
\hline & Ángulo externo lóbulo $\left(^{\circ}\right)$ & AngextLAQ & \\
\hline & $\begin{array}{l}\text { Longitud sépalos } \\
\text { fructíferos }(\mathrm{mm})\end{array}$ & LongSF & \\
\hline & $\begin{array}{l}\text { Ancho 1er tercio de } \\
\text { sépalo fructífero }(\mathrm{mm})\end{array}$ & AnchoSF1/3 & \\
\hline & $\begin{array}{l}\text { Ancho } 2 \text { do tercio de } \\
\text { sépalo fructífero (mm) }\end{array}$ & AnchoSF2/3 & \\
\hline & $\begin{array}{l}\text { Ancho 3er tercio de } \\
\text { sépalo fructífero }(\mathrm{mm})\end{array}$ & AnchoSF3/3 & \\
\hline
\end{tabular}


Bol. Soc. Argent. Bot. 55 (3) 2020

Tabla 2. Variables vegetativas significativamente diferentes $(p<0,05)$ entre los Grupos 1 y 2 , según la prueba de Kruskal-Wallis.

\begin{tabular}{|llc|}
\hline \multicolumn{1}{c}{ Variables } & \multicolumn{1}{c|}{ Abreviación } & p \\
Alt & Altura & $<0,0001$ \\
PromPer & Promedio perímetro & $<0,0001$ \\
LongH & Longitud de hoja & $<0,0001$ \\
AnchoH & Ancho de hoja & $<0,0001$ \\
LongLamH & Longitud de lámina & $<0,0001$ \\
LongPecH & Longitud de pecíolo & $<0,0001$ \\
AnchoPecH & Ancho de pecíolo & $<0,0001$ \\
HábCrec & Hábito de crecimiento & $<0,0001$ \\
FH_4 & Forma de hoja (elíptico lanceolada) & $<0,0001$ \\
BH_1 & Base de hoja (aguda) & $<0,0001$ \\
CH & Consistencia de hoja & $<0,0001$ \\
DiNH & Ángulo de divergencia de nervaduras secundarias & $<0,0001$ \\
DeNH & Densidad de nervaduras en hoja & $<0,0001$ \\
PEH_1 & Pilosidad en envés de hoja (escasa) & $<0,0001$ \\
\hline PEH_3 & Pilosidad en envés de hoja (glabra-subglabra) & $<0,0001$ \\
AbPNEH_2 & Abundancia de pilosidad en nervaduras de envés (baja) & $<0,0001$ \\
\hline AbPNEH_3 & Abundancia de pilosidad en nervaduras de envés (media) & $<0,0001$ \\
PPH & Grado de pilosidad de pecíolo & $<0,0001$ \\
PO_4 & Pilosidad de ócrea (muy pilosa) & $<0,0001$ \\
FH_1 & Forma de hoja (aovada) & 0.001 \\
BH_7 & Base de hoja (obtuso redondeada) & 0.001 \\
NHH & Intensidad de resaltado de nervaduras en haz & 0.001 \\
PO_2 & Pilosidad ócrea (mediana) & 0.005 \\
LongO & Longitud de ócrea & 0.029 \\
\hline
\end{tabular}




\section{Á. Tessore et al. - Variabilidad morfológica de Ruprechtia apetala en Argentina}

Tabla 3. Variables reproductivas para Grupos 1 y 2 .Para cada una se detallan valores de media y desvío estándar (D.E.). Valores de variables cualitativas refieren a frecuencias entre 0 y 1.

\begin{tabular}{|c|c|c|c|c|c|}
\hline \multirow[b]{2}{*}{ Variables reproductivas } & \multirow[b]{2}{*}{ Abreviaturas } & \multicolumn{2}{|c|}{ GRUPO 1} & \multicolumn{2}{|c|}{ GRUPO 2} \\
\hline & & $\begin{array}{c}\text { Media/ } \\
\text { Frecuencia }\end{array}$ & $\begin{array}{l}\text { Desvío } \\
\text { Estándar }\end{array}$ & $\begin{array}{c}\text { Media/ } \\
\text { Frecuencia }\end{array}$ & $\begin{array}{l}\text { Desvío } \\
\text { Estándar }\end{array}$ \\
\hline Longitud aquenio (mm) & LongAQ & 7.95 & 0.65 & 8.04 & 0.80 \\
\hline Ancho aquenio (mm) & AnchoAQ & 3.78 & 0.14 & 3.78 & 0.28 \\
\hline Longitud pedicelo aquenio (mm) & LongPAQ & 3.74 & 0.61 & 3.95 & 1.12 \\
\hline Largo lóbulo aquenio (mm) & LargoLAQ & 2.24 & 0.11 & 2.22 & 0.18 \\
\hline Ancho lóbulo aquenio (mm) & AnchoLAQ & 2.53 & 0.11 & 2.47 & 0.21 \\
\hline Ángulo interno lóbulo aquenio $\left({ }^{\circ}\right)$ & AngintLAQ & 118.89 & 5.41 & 119.25 & 6.91 \\
\hline Ángulo externo lóbulo aquenio $\left({ }^{\circ}\right)$ & AngextLAQ & 102.65 & 14.66 & 112.83 & 26.36 \\
\hline Longitud sépalos fructíferos (mm) & LongSF & 26.04 & 2.92 & 25.59 & 3.74 \\
\hline Ancho sépalo fructífero (mm) & AnchoSF & 4.21 & 0.45 & 4.24 & 0.59 \\
\hline Ancho 1er tercio de sépalo fructífero $(\mathrm{mm})$ & AnchoSF1/3 & 4.46 & 0.80 & 4.28 & 0.80 \\
\hline Ancho 2do tercio de sépalo fructífero (mm) & AnchoSF2/3 & 3.53 & 0.63 & 3.31 & 0.70 \\
\hline Ancho 3er tercio de sépalo fructífero (mm) & AnchoSF3/3 & 3.62 & 1.07 & 3.55 & 0.92 \\
\hline Forma de apice de sépalos fructíferos (aguda) & FASF_1 & 0.46 & - & 0.19 & - \\
\hline Forma de apice de sépalos fructíferos (obtusa) & FASF_2 & 0.06 & - & 0.12 & - \\
\hline Forma de apice de sépalos fructíferos (redondeada) & FASF_3 & 0.46 & - & 0.65 & - \\
\hline Forma de apice de sépalos fructíferos (retusa) & FASF_4 & 0.02 & - & 0.04 & - \\
\hline Grado de pilosidad de sépalos fructíferos (muy pilosos) & PSF_1 & 0.79 & - & 0.56 & - \\
\hline Grado de pilosidad de sépalos fructíferos (pilosos) & PSF_2 & 0.14 & - & 0.37 & - \\
\hline Grado de pilosidad de sépalos fructíferos (poco pilosos) & PSF_3 & 0.07 & - & 0.07 & - \\
\hline Grado de pilosidad de pedicelo (glabro) & PP_1 & 0.07 & - & 0.07 & - \\
\hline Grado de pilosidad de pedicelo (glabro-piloso) & PP_2 & 0.64 & - & 0.30 & - \\
\hline Grado de pilosidad de pedicelo (muy piloso) & PP_3 & 0.29 & - & 0.62 & - \\
\hline Coloración de sépalos fructíferos (cobrizo) & CSF_1 & 0.00 & - & 0.11 & - \\
\hline Coloración de sépalos fructíferos (marrón oscuro) & CSF_2 & 0.00 & - & 0.00 & - \\
\hline Coloración de sépalos fructíferos (marrón pálido) & CSF_3 & 0.29 & - & 0.30 & - \\
\hline Coloración de sépalos fructíferos (naranja cobrizo) & CSF_4 & 0.00 & - & 0.23 & - \\
\hline Coloración de sépalos fructíferos (naranja pálido) & CSF_5 & 0.14 & - & 0.36 & - \\
\hline Coloración de sépalos fructíferos (rosa pálido) & CSF 6 & 0.57 & - & 0.00 & - \\
\hline
\end{tabular}


